\title{
COALICIONES SIN PARTIDOS. LA CIUDAD DE Buenos Aires lUeGo de LA CRISIS De 2001
}

\author{
Sebastián Mauro \\ sebasmauro@hotmail.com \\ CONICET - Instituto de Investigaciones Gino Germani \\ Universidad de Buenos Aires
}

La crisis argentina de fines de 2001 llevó al colapso del sistema de partidos dentro de la Ciudad Autónoma de Buenos Aires, dejando un espacio político fragmentado y volátil que se prolongó hasta bien entrado el ciclo político posterior. Los partidos principales del distrito se habían disuelto o fracturado y el número de alianzas electorales aumentó, convirtiéndose en la principal herramienta para la reconstrucción de los partidos. El artículo examina las características de estas coaliciones y se pregunta cuáles fueron las consecuencias para la institucionalización del sistema de partidos y para la reconstrucción de los vínculos de representación, del mismo modo se comparan las estrategias de coalición de las principales fuerzas políticas en las elecciones de 2003 y 2007.

Palabras clave: Partidos políticos, coaliciones, representación política, Buenos Aires.

\section{Coalitions Without parties: The city of Buenos AIRES SINCE THE CRISIS OF 2001}

The Argentine crisis in late 2001 led to the collapse the party system within the Autonomous City of Buenos Aires, leaving a fragmented and volatile political arena which lasted well into the succeeding political cycle, while the district's main parties either dissolved or fractured, the number of electoral alliances increased, becoming the principal tool for party construction. The paper examines the characteristics of these coalitions and asks what were the consequences for the institutionalization of the party system and for the reconstruction of representative links; the article compares coalitional strategies of the main political forces in the 2003 and 2007 elections in the City of Buenos Aires.

Keywords: Political parties, coalitions, political representation, Buenos Aires. 


\section{INTRODUCCION ${ }^{1}$}

La crisis argentina de fines de 2001 significó el quiebre del sistema bipartidista vigente desde 1983, con el predominio del Partido Justicialista (PJ) y la Unión Cívica Radical (UCR). La ruptura del bipartidismo, sin embargo, no impactó de igual forma a los dos partidos predominantes ni impulsó la emergencia de nuevos y más representativos partidos políticos. La interrupción de la presidencia de Fernando de la Rúa (en un gobierno de coalición entre la UCR y el Frente País Solidario, FREPASO), condujo al derrumbe electoral del radicalismo y a la disolución de las terceras fuerzas políticas recientemente formadas. Contrariamente a la fragmentación y declive de los partidos del polo no peronista, el PJ logró, luego de la crisis, incrementar su poder institucional, renovar su liderazgo nacional y revalidar sus vínculos con la ciudadanía.

Los datos sobre la crisis del sistema partidario son elocuentes: el número efectivo de partidos en elecciones presidenciales varió de 2 en 1983 a 5,7 en 2003, mientras que el número efectivo de bloques en la Cámara de Diputados de la Nación pasó de un promedio de 2,5 durante la década de los '80 a un promedio de 4,4 entre 2003 y 2011. También es elocuente el impacto diferencial de dicha crisis entre el PJ y la UCR. En primer lugar, el radicalismo pasó de un caudal electoral de 51,7\% en las elecciones presidenciales de 1983 a 2,3\% en las de 2003 y a 16,9\% en las de 2007, mientras que el peronismo pasó de 40,2\% en 1983 a 45\% en 2007. En segundo lugar, la representación parlamentaria de la UCR en la Cámara de Diputados descendió del 49\% durante el período 1983-1989 al 17\% en el período 2003-2007, mientras que el PJ pasó de $40 \%$ en el primer período a $50 \%$ en el segundo.

El quiebre del bipartidismo por el derrumbe de uno de sus componentes es un diagnóstico compartido por la literatura para caracterizar la política argentina a nivel nacional (Torre, 2003; Calvo y Escolar, 2005), pero no se ajusta a la realidad de los distritos subnacionales, donde, en muchos casos, la UCR logró retener una importante cuota de poder institucional, profundizando la tendencia a la desnacionalización o federalización del sistema partidario (Gibson y Suárez Cao, 2010).

El único distrito subnacional donde no se ha impuesto este patrón de competencia interpartidaria es la Ciudad Autónoma de Buenos Aires (capital de la República y segundo distrito electoral más populoso), donde la movilización social generalizada

Una versión preliminar de este trabajo fue presentada en el XXI Congreso Mundial de Ciencia Política, ¿Descontento Global? Dilemas de cambio, Santiago de Chile, 12 al 16 de julio de 2009. 
de 2001 generó el colapso del sistema partidario en su conjunto. Una serie de indicadores permiten dar cuenta del profundo quiebre que significó la crisis de 2001 para el sistema político porteño.

En primer lugar, es posible observar cómo los principales partidos del distrito (PJ, UCR, FREPASO y otros partidos menores) fueron marginados de la competencia política luego de la crisis: en las elecciones para diputados nacionales del año 2003, los principales partidos sumados concentraron apenas el 3,3\% de los votos; mientras que en los otros distritos subnacionales, los partidos tradicionales continuaban representando a más del 50\% del electorado (Bril Mascarenhas, 2007). La marginación de los partidos tradicionales no significó un proceso lineal de renovación, sino la conformación de una escena fragmentaria y volátil, en la que se sucederían sellos partidarios inestables alrededor de líderes personalistas.

Esta nueva dinámica se expresó en la proliferación de partidos competitivos en el sistema político. Si se observa el número efectivo de partidos para todas las categorías, podrá identificarse la explosión del número de partidos: en la categoría Jefe de Gobierno pasó de 2,8 (en las elecciones de 2000) a 6 (en 2003); en la categoría legisladores locales pasó de 2,7 (1997) a 10,3 (2003); y, finalmente, en la categoría diputados nacionales pasó de 2,3 (1997) a 10 (2007). La volatilidad del sistema impactó en la Legislatura local, donde las coaliciones parlamentarias se fragmentaron profundamente: mientras que entre 1997 y 2001 funcionó con cinco bloques, entre 2002 y 2003 se fracturó en alrededor de 25 bloques.

Curiosamente, a pesar de la gran volatilidad del sistema político y de sus efectos disolventes para la formación de coaliciones parlamentarias duraderas, la baja institucionalización del sistema partidario redundó en una multiplicación de alianzas electorales, que se convirtieron en la principal herramienta de construcción partidaria utilizada por líderes personalistas. ¿Qué características asumen estas coaliciones y qué consecuencias implican para la institucionalización del sistema partidario y para la reconstrucción de los lazos representativos? El artículo describe y compara las estrategias coalicionales de las principales fuerzas políticas en las elecciones de 2003 y 2007 en la Ciudad de Buenos Aires, de renovación de autoridades ejecutivas y legislativas locales. La hipótesis que guía el análisis es que luego de la crisis se instaló un formato partidario basado en la articulación variable entre líderes con popularidad en la opinión pública, fragmentos de estructuras territoriales y cuadros políticos y técnicos en disponibilidad. Estos nuevos formatos partidarios asumieron la forma institucional de alianzas electorales y coaliciones políticas circunstanciales, 
de acuerdo a las posibilidades de establecer vínculos de representación con una ciudadanía que se comporta de manera volátil y selectiva.

\section{Características salientes de la Ciudad de Buenos Aires COMO DISTRITO}

La ciudad capital de la República Argentina es el segundo distrito electoral más grande del país: alberga alrededor del 10\% del total del padrón nacional, siendo superada sólo por la Provincia de Buenos Aires. La concentración del electorado determina una representación importante en la Cámara de Diputados de la Nación, donde ocupa cerca del 10\% de los escaños. En la ciudad residen las autoridades estatales nacionales y las principales instituciones financieras, educativas, comerciales y sanitarias, así como los mayores centros culturales, deportivos y de esparcimiento de la Argentina, lo que instituye a Buenos Aires como el principal centro político, económico y cultural del país. En consecuencia, la Ciudad ha constituido, históricamente, el polo de atracción de casi todas las organizaciones políticas y sociales con pretensión de posicionarse en el nivel nacional ${ }^{2}$.

La concentración de recursos políticos, económicos y sociales en el territorio de Buenos Aires ha sido uno de los problemas constitutivos de la fundación del Estado argentino, cuya solución fue la forzada federalización de la Ciudad, separada del territorio bonaerense para convertirse en la Capital de la República, lo que constituyó uno de los pilares de la organización nacional hacia 1880. Si bien la posición de la Ciudad como el centro del poder político y económico del país se vio reafirmada, el carácter de territorio federal restringía toda forma de autogobierno: su intendente era designado por el Presidente de la Nación, mientras que la sanción de leyes recaía en el Congreso Nacional, que delegaba sólo los asuntos municipales en el Concejo Deliberante, única instancia representativa del distrito. Ello dio a la Ciudad una identidad particular fuerte, constituyéndose en sede de fuerzas renovadoras (como el socialismo y la UCR a principios del siglo XX) y opositoras a los gobiernos nacionales (como sucedió durante el peronismo a mediados de siglo). Luego de la recuperación democrática en 1983, la Ciudad favoreció a fuerzas políticas ajenas al

2 La ciudad es la sede del $90 \%$ de los partidos políticos nacionales inscriptos, así como del $60 \%$ de las organizaciones de la Sociedad Civil con alcance nacional. Por otra parte, la ciudad es el principal centro de producción y circulación en el campo mediático, tanto en periódicos como en producción televisiva. 
bipartidismo, como el Partido Intransigente, la Unión de Centro Democrática y el FREPASO.

A fines del siglo XX se inició el proceso de “autonomización”, por el cual la Ciudad mantenía su estatuto de sede de las autoridades políticas nacionales, pero en calidad de Ciudad Autónoma. Con la autonomía fue sancionado un conjunto de instituciones políticas y se articuló un sistema político estrictamente local. La Ciudad Autónoma de Buenos Aires es gobernada por un Jefe de Gobierno elegido de manera directa en doble vuelta -excepto que la fórmula ganadora obtenga más del $50 \%$ de los votos en la primera vuelta-. El Poder Legislativo local consiste en un solo cuerpo de 60 miembros que se renueva por mitades cada dos años. Los legisladores son electos en distrito único, a través de listas cerradas y bloqueadas, por el sistema D'Hont, sin umbral para la asignación de bancas, por un mandato de cuatro años.

A diferencia del rasgo saliente del resto de los distritos subnacionales, la Ciudad se destaca por la ausencia absoluta de sesgos mayoritarios o partidarios en su normativa electoral (Calvo y Escolar, 2005). El uso de la fórmula D'Hont sin umbral para la asignación de bancas en un distrito de magnitud alta ha definido una proporcionalidad casi perfecta entre votos y bancas ${ }^{3}$. Otro tanto puede decirse de la legislación electoral para la designación del Ejecutivo local: con requisitos más exigentes que los de la legislación nacional para que un candidato se imponga en el primer turno, la normativa electoral ha facilitado la dispersión de la oferta partidaria en primera vuelta, haciendo del ballotage una práctica recurrente.

Asimismo, el sistema electoral no impone ninguna restricción a una práctica que se volvió habitual en la última década: la presentación de distintas listas legislativas en apoyo a una única fórmula al ejecutivo. Ello ha favorecido, en un contexto de desagregación partidaria y vaciamiento de la vida interna de los partidos, la práctica de multiplicar las candidaturas para resolver potenciales conflictos en el proceso de nominación, generando una oferta electoral piramidal que funciona como una especie de sistema de "neolemas", donde diferentes listas legislativas aportan al triunfo de un único candidato al ejecutivo local. Este fenómeno, por su parte, incide en el acceso de los partidos a la Legislatura, en tanto las boletas para cargos legislativos y ejecutivos se encuentran unidas en el cuarto oscuro, facilitando el arrastre de votos que puede ejercer la figura popular del candidato a Jefe de Gobierno.

\footnotetext{
El índice de Loosemory-Hanby (que expresa cuán disímil es la asignación de bancas respecto de los votos obtenidos, según el cual 1 señala la desproporcionalidad total y 0 , la proporcionalidad pura) para la ciudad en el período 1997-2007 fue de 0,1.
} 
Estos elementos del sistema electoral parecen explicar la naturaleza de la volatilidad en el comportamiento electoral y de la fragmentación partidaria. Sin embargo, es necesario señalar que el proceso de descongelamiento del sistema partidario porteño ya se había iniciado con anterioridad a la declaración de la autonomía porteña, y que el marco institucional fue el resultado de ese proceso antes que su origen. La Constitución de la Ciudad Autónoma, así como sus principales leyes, fueron redactadas por cuerpos legislativos compuestos en gran medida por fuerzas políticas renovadoras, emergentes del descontento ciudadano con los partidos tradicionales y promotoras de una legislación permeable a la renovación política, inversa al tipo de reformas que primaron en el resto de los distritos.

En el próximo apartado analizaremos cómo estas características salientes de la Ciudad de Buenos Aires como distrito electoral incidieron en el contexto de crisis política emergente de la protesta social de 2001, y fueron instrumentalizados por los actores políticos para reconfigurar un sistema político basado en la inestabilidad de las alianzas partidarias.

\section{LA CRISIS Y LA REORGANIZACIÓN DE ALIANZAS INESTABLES}

La crisis política de fines de 2001 afectó particularmente a la ciudad de Buenos Aires. En primer lugar, porque la ciudad fue el epicentro de las protestas sociales que obligaron la salida del gobierno de la Alianza y boicotearon los intentos posteriores de normalización. En segundo lugar, porque la interrupción de la presidencia de Fernando de la Rúa disolvió la coalición entre dos partidos con fuerte raigambre en la ciudad: la UCR y el FREPASO, así como eliminó de la política a las dos figuras más representativas del distrito (el propio De la Rúa y quien fue su vicepresidente, Carlos “Chacho” Álvarez).

Estos elementos condujeron al colapso completo del sistema partidario porteño, a contramano de la "normalización" política y económica que había iniciado el gobierno provisional del peronista Eduardo Duhalde. La presidencia de Duhalde lidió exitosamente con los problemas económicos y logró estabilizar el sistema político nacional, aunque las elecciones presidenciales de 2003 demostraron hasta qué punto las solidaridades políticas se encontraban fragmentadas: seis candidatos (tres del polo peronista y tres provenientes del radicalismo) aparecían como figuras relativamente competitivas, lo que generaba un escenario de incertidumbre sobre el futuro del país. Frente a esta incertidumbre, los gobiernos provinciales respondieron inten- 
tando desacoplar la dinámica política nacional de la distrital, con la consecuente profundización del proceso de federalización de la política (dicho fenómeno se agravaba por el hecho de que las elecciones legislativas nacionales se realizarían, de manera excepcional, concurrentemente con las elecciones distritales, en lugar de coincidir con la fecha de los comicios a Presidente y vicepresidente). En la ciudad, donde la crisis era más pronunciada, el escenario preelectoral comenzó a perfilarse a partir de figuras personalistas que se autoproclamaron como candidatos, convocando a diversos sectores a formar coaliciones con un amplio margen de maniobra.

Un potencial polo de atracción era el mandatario local,Aníbal Ibarra, quien aparecía como capaz de representar una propuesta de centroizquierda, identificada con la retórica del ex FREPASO pero sin haber sucumbido ante el fracaso de la Alianza.A pesar de la situación crítica que enfrentó el gobierno porteño a fines de 2001, con el desorden de la protesta generalizada en sus calles y la disolución de la coalición de gobierno, Ibarra logró sostener el control del Ejecutivo, asegurando la solvencia de las cuentas públicas y garantizando canales de diálogo con los colectivos de protesta emergentes, a partir de la implementación descentralizada de políticas públicas focalizadas.

La capacidad de Ibarra como núcleo de un espacio de centroizquierda, sin embargo, no fue producto inmediato de estos factores, sino que requirió de múltiples usos instrumentales de la legislación electoral (especialmente, del calendario) para instalar una escena polarizada con una opción de centroderecha.

Poco tiempo antes de los comicios presidenciales, el jefe de gobierno adelantó la convocatoria a elecciones locales, dejando a los actores políticos sin tiempo de formar coaliciones y nominar candidatos ${ }^{4}$. De este modo, aislaba la competencia local de la situación nacional: ante la incertidumbre sobre qué alineamientos políticos nacionales resultarían de la elección presidencial, cualquier negociación de candidaturas en el distrito se volvía provisoria. Asimismo, la superposición con la campaña electoral nacional complicaba a los candidatos presidenciales con buena imagen en el electorado porteño para competir personalmente en las dos arenas. Por último, con el adelantamiento de las elecciones, forzaba la renuncia de los precandidatos con menos reconocimiento entre el público. De este modo, Ibarra intentaba controlar las consecuencias que la dispersión partidaria podía ocasionarle a su candidatura, frente

\footnotetext{
4 Los comicios estaban previstos para septiembre u octubre, pero Ibarra anunció en el inicio de las sesiones ordinarias de la Legislatura su convocatoria al 8 de junio. Según este calendario, las candidaturas debían oficializarse el 18 de abril, nueve días antes de las elecciones presidenciales.
} 
a las buenas perspectivas que le auguraban los sondeos preelectorales al empresario y dirigente futbolístico Mauricio Macri, quien había hecho pública su voluntad por competir en el proceso electoral.

Sin embargo, esta estrategia fue frustrada por la Justicia electoral, que obligó al Jefe de Gobierno a postergar los comicios por los múltiples problemas organizativos ocasionados por el adelantamiento. En los dos meses que transcurrieron entre el proceso electoral frustrado y la celebración efectiva de las elecciones se celebraron los comicios presidenciales y el campo político entró en una rápida reorganización, que contribuiría, casi accidentalmente, a la ambición reeleccionista del mandatario porteño.

El polo de dicha reorganización fue el nuevo Presidente. Luego de acceder al cargo con el 22\% de los votos, Kirchner procuró construir un liderazgo de popularidad que superó los logros del gobierno duhaldista en materia de estabilización de la economía, y que, al mismo tiempo, amplió su agenda con una promesa de recambio político. La temprana implementación de políticas que recuperaban algunas de las más importantes demandas sociales, como el tratamiento no represivo de la protesta social, el impulso a la renovación de la Corte Suprema de Justicia o la activa política sobre derechos humanos, señaló ante la opinión pública la voluntad de implementar una agenda que ampliaba los límites de los gobiernos precedentes. Estas políticas hicieron verosímil la convocatoria del Presidente hacia los actores de la centroizquierda del arco político, que atrajo a dirigentes políticos (del FREPASO y del PJ disidente), a las dos centrales gremiales y a parte del movimiento piquetero. La formación de esta nueva coalición de gobierno, que incluía y trascendía al peronismo, recibió el nombre de transversalidad, y tenía una doble significación. En el plano estratégico, el kirchnerismo ganaba autonomía frente al liderazgo de Duhalde y al poder de veto de los gobernadores peronistas. Pero dicha estrategia se inscribía en un proyecto de construcción identitaria, que, al mismo tiempo, producía una diferenciación respecto de las políticas de la década precedente, y una identificación con valores de la centroizquierda, en sintonía con la evolución política del resto de los países de la región.

La aparición de Kirchner en la escena nacional no sólo funcionó como polo de atracción para dispersos actores de la izquierda peronista y del FREPASO, sino que también instaló en la opinión pública un clima de "cambio de época", cuyo sentido se anclaba en las promesas de inclusión social, revisión de las políticas neoliberales y promoción de los derechos humanos. Para el perfil de Ibarra, la convocatoria de Kirchner y el sentido que éste asignaba a la renovación política constituían una oportu- 
nidad de volver a presentarse como parte de un espacio progresista. Ello lo ubicaba en mejor posición que Macri, asociado a la figura del ex presidente Carlos Menem.

Más allá de esta sintonía en el discurso político, Kirchner contribuyó a la instalación de Ibarra al forzar la renuncia de sus dos precandidatos, que fueron designados en el gabinete nacional. Esta contribución obedecía al cálculo, validado por los sondeos de opinión, de que ninguno de los dos estaba en condiciones de disputar el gobierno porteño. Frente a ello, Ibarra aparecía como un aliado seguro del gobierno y una mejor opción que Macri, potencial opositor.

Sin embargo, dichas contribuciones del kirchnerismo hacia la candidatura ibarrista no eran suficientes para garantizarle al Jefe de Gobierno una buena performance electoral. Antes que presentarse sin mediaciones como el candidato del Presidente, Ibarra requería de la concurrencia de un abanico heterogéneo de actores, que incluía a los partidos Socialista (PS) y Afirmación por una República Igualitaria (ARI) en un espacio progresista, que atrajera tanto al electorado peronista como no peronista y pudiera competir con la popularidad de Macri.

Ubicado como principal referente de un abanico de fuerzas políticas, con el apoyo de líderes nacionales como Kirchner, Elisa Carrió y Alfredo Bravo, Aníbal Ibarra presentó su nuevo sello partidario, Fuerza Porteña, incorporando al Frente Grande (ex FREPASO) en coalición con otros partidos ${ }^{5}$. Acompañado por el Ministro de Cultura de la Ciudad, el justicialista Jorge Telerman, la candidatura al ejecutivo local funcionó como el vértice de una pirámide de listas para cargos legislativos. Habilitando la coexistencia de diferentes listas que apoyaban al mismo candidato al ejecutivo, Ibarra resolvía la negociación por la definición de candidaturas (compleja por la ausencia de mecanismos internos para la resolución de conflictos y por la dificultad de comparar el peso relativo de cada actor dentro de la alianza), exteriorizando la disputa interna a la elección general, como una suerte de "neolemas". La estrategia se veía favorecida por el sistema electoral porteño, perfectamente proporcional y sin umbrales para la asignación de bancas. Además, producto de la iniciativa de revocar todos los mandatos, la Legislatura porteña se renovó completamente por tercera vez consecutiva, lo que permitía a fuerzas políticas menores acceder a alguna banca con un menor caudal de votos. Por otro lado, la excepcional concurrencia de las eleccio-

\footnotetext{
Los partidos que formaban parte de la alianza Fuerza Porteña eran: FG, ARI, PS, PAIS, Partido Intransigente, Partido de laVictoria (el nuevo sello electoral del Presidente), GESTA (el sello electoral de Rafael Bielsa), y Memoria y Movilización Social (el sello electoral del dirigente justicialista Juan Carlos Dante Gullo, expulsado del ARI a inicios de 2003 por diferencias con Elisa Carrió).
} 
nes a diputados nacionales facilitó la negociación ampliando el número de lugares a distribuir: Ibarra privilegió sumar bancas en la legislatura local, dejando a sus socios sumarlas en el Congreso.

En el caso de las listas para diputados nacionales, Fuerza Porteña distribuyó las candidaturas entre los principales socios de Ibarra: el kirchnerismo, el ARI, el Socialismo y sectores gremiales. Además, el jefe de gobierno aceptó que otras dos listas de candidatos a diputados nacionales apoyaran su candidatura, una de ellas encabezada por un periodista vinculado al kirchnerismo, la otra por una funcionaria porteña de extracción radical. Las listas de candidatos a la legislatura porteña replicaban el loteo de espacios políticos, incorporando a la lista principal (Fuerza Porteña) un puñado de candidatos leales al jefe de gobierno.

Por su parte, Mauricio Macri, presentó su nuevo partido, Compromiso para el Cambio (CPC), en alianza con una pluralidad de partidos menores referenciados en la centroderecha del arco político ${ }^{6}$. Aunque también construyó su coalición en forma piramidal, presentó sólo una lista de candidatos a diputados nacionales, compuesta por los referentes del peronismo porteño no alineados con el liderazgo de Kirchner. Éstos se alternaban con referentes de otros partidos, especialmente del radicalismo y del minoritario y conservador Partido Demócrata.

Para la legislatura local, Macri recurrió a la estrategia de externalizar la competencia entre las facciones, y presentó cuatro listas de candidatos. El sello CPC (en alianza con la conducción del peronismo porteño y el Partido Demócrata Progresista) promocionaba la candidatura de jóvenes cuadros técnicos con mínima trayectoria política, seguidos por figuras de heterogénea inscripción partidaria: radicales, peronistas y de fuerzas conservadoras menores. Otras dos listas (una denominada Frente de la Esperanza Porteña y otra, Movimiento Generacional), agrupaban a diferentes fragmentos del peronismo porteño que se habían alejado tanto de la conducción partidaria local como nacional. Finalmente, la cuarta lista de candidatos a la Legislatura agrupaba al resto de los cuadros políticos de las pequeñas fuerzas conservadoras que apoyaban a Macri.

Otras dos figuras de renombre lograron aglutinar a diferentes actores políticos, y construir tras de sí alianzas. El diputado de izquierda Luis Zamora, de sorprendente

\footnotetext{
A nivel de diputados nacionales, la alianza CPC incluía a los partidos CPC, Demócrata, Federal, Acción por la República (sello del ex ministro de economía Domingo Cavallo), Autonomista, Nacionalista Constitucional, Todos por los Jubilados y Popular Cristiano.
} 
performance en 2001, abandonó la estrategia abstencionista que promovió para las presidenciales, y promovió la candidatura de referentes del movimiento asambleario. La dirigente de centroderecha Patricia Bullrich, por su parte, formó una alianza con el ex ministro de la Alianza Ricardo López Murphy, que había logrado una buena performance electoral en las elecciones presidenciales y necesitaba sumar poder institucional.

La lista de elencos políticos que compitieron en las elecciones de agosto de 2003 se completaba con un elevado número de partidos menores, algunos de larga trayectoria y otros de reciente inscripción. Se presentaron 31 fórmulas para el ejecutivo, 33 listas para diputados nacionales y 38 para legisladores, un número que más que duplicaba a las cantidades de las elecciones anteriores ${ }^{7}$, poniendo en evidencia la profunda crisis del sistema político local.

La campaña electoral giró en torno a la polarización entre una vieja política corrupta y una nueva política (Mauro, 2005). En este sentido, la presentación de la coalición que sostenía al Jefe de Gobierno volvía verosímil la idea de un frente de centroizquierda que daba batalla a las aspiraciones personales de uno de los beneficiarios de las políticas de los '90: mientras que detrás de Ibarra se alineaba una serie de actores identificados con una retórica progresista, la propuesta de Macri era presentada como la aventura personal de un empresario corrupto y demagógico. Identificada con la década pasada, la corrupción era entendida como apropiación de los bienes de todos por parte de algunos, entendiendo como progresista la protección de lo público por parte del Estado. Si bien esta concepción podía evocar vagamente una demanda de inclusión social, lo público era interpretado como un espacio neutral, igualmente de todos e idealmente ajeno a la lucha política. Por esta operación, la centroderecha aparecía ligada a intereses particulares en detrimento de lo público, pero al precio de que el progresismo apareciera vaciado de las reivindicaciones históricas de la izquierda democrática.

Del lado del macrismo, el intento por desmontar este principio de lectura también tomó como eje la divisoria entre vieja y nueva política, pero entendiendo el carácter corrupto de la vieja política en los términos de una "politiquería vacía”, que promete para acceder al poder y luego no se esfuerza por solucionar los problemas de la gente. En este sentido, CPC exaltó (ya desde el nombre del sello) la inserción de

\footnotetext{
7 En 1997 participaron 18 listas de candidatos a legisladores porteños, y 17 en las elecciones de 2000. En las elecciones a diputados nacionales de 2001, se presentaron 19 listas por la Ciudad de Buenos Aires.
} 
recursos humanos ajenos a la política (y, automáticamente, a su corrupta situación) dispuestos a llevar adelante los cambios que el público esperaba.

De esta forma, tanto el progresismo como el sector identificable con la centroderecha, compartían un modelo de diferenciación con el pasado reciente (la "vieja política"), caracterizado por la debacle y la corrupción, entendida esta última como obstáculo para la plenitud de todos en beneficio de algunos. Estas líneas discursivas se convirtieron en ejes de la división ideológica del campo político porteño, delineando afinidades identitarias entre los sectores que participaban de las alianzas electorales en el período siguiente.

En las elecciones del 24 de agosto, Mauricio Macri se impuso con el 37,5\% de los votos, el resultado más bajo en las tres elecciones ejecutivas de la Ciudad, por lo que debió enfrentar una segunda vuelta electoral contra la fórmula Ibarra-Telerman, que había obtenido el 33\% de los votos. En la nueva competencia, se puso de manifiesto el difundido rechazo a la figura de Macri, revelando la eficacia de la construcción discursiva de Ibarra. El Jefe de Gobierno, finalmente, fue reelecto por el 53,5\% de los votos, frente al 46,5\% de la fórmula de CPC.

No obstante, el macrismo conquistó cinco bancas en la Cámara de Diputados de la Nación, y, sumadas sus cuatro listas de candidatos, obtuvo 23 bancas en la Legislatura local. El ibarrismo, en cambio, conquistó cuatro bancas para el Congreso Nacional, y 21 bancas en la Legislatura porteña. Zamora y Bullrich lograron instalarse como líderes de espacios políticos significativos en el distrito, conquistando 8 y 6 bancas en la Legislatura local respectivamente.

\section{LA FALLIDA CONSOLIDACIÓN DEL NUEVO SISTEMA PARTIDARIO}

De este modo, se produjo un resultado ambiguo: mientras Aníbal Ibarra había logrado la reelección y había validado su discurso de impugnación a la figura de Macri (cuyo nivel de rechazo entre el electorado se puso de manifiesto en el ballotage), el ingeniero había logrado reunir un numeroso grupo de legisladores, potencialmente más poderoso que el resto de los fragmentados actores que habían ingresado al reparto de poder institucional.

El desequilibrio producido por esta distribución del poder institucional se profundizaría progresivamente, a partir de la fragmentación y del realineamiento de los diferentes sectores que participaron de los armados electorales. De este modo, a los 
pocos meses de asumir, los diputados electos por las tres listas ibarristas se dividieron en doce bloques diferentes, mientras que los legisladores zamoristas se dividieron en cuatro bloques y los legisladores que apoyaron a Patricia Bullrich se fragmentaron en otros tres. Contrariamente a este proceso, los legisladores que respondían a Mauricio Macri se reagruparon en un único y numeroso bloque, aunque con el tiempo también sufrieron un proceso de fractura y erosión, aunque menos profundo que en el resto de los espacios políticos. De este proceso de fragmentación y realineamiento emergerían nuevos protagonistas de la política porteña: el kirchnerismo y el ARI formarían bloques propios con gran capacidad de negociación, cada vez más independientes de la figura del jefe de gobierno.

Frente a la inestabilidad de las alianzas, tanto el oficialismo local como la oposición recurrieron a reafirmar los discursos polarizadores sobre la corrupción, en el intento de mantener líneas de división organizadoras del campo político que facilitaran la rehabilitación de las alianzas ante coyunturas específicas o frente a temas de debate divisivos. Esta dinámica funcionó para el tratamiento de cuestiones como la reforma del Código Contravencional de la Ciudad o en debates sobre educación sexual, casos en los que el bloque macrista desarrolló activamente una agenda conservadora, mientras que el resto de los actores se limitó a contener o moderar los intentos de reforma sobre la legislación progresista vigente.

Esta dinámica encontró un nuevo nivel luego del incendio de un local durante un recital de rock a fines de 2004, en el que murieron 194 jóvenes y miles resultaron heridos. La movilización social emergida de la tragedia generó una crisis en la coalición de gobierno ibarrista, que casi inmediatamente perdió el apoyo de los sectores progresistas que habían participado de la alianza electoral Fuerza Porteña en 2003. Estos sectores (en particular el ARI) pasaron a formar parte de una avanzada opositora liderada por el macrismo y apoyada sistemáticamente por los partidos de izquierda (fragmentos de la coalición que lideró Luis Zamora en 2003), que amenazaba con forzar la renuncia o la destitución del mandatario local. La crisis obligó a Ibarra a reorientar su coalición de gobierno recostándose en el apoyo del peronismo (tanto kirchnerista como no kirchnerista) y de otros sectores políticos menores, desdibujando la identidad ideológica construida dos años antes. Frente a esta evolución, Ibarra denunció un intento de golpe institucional por parte de la centroderecha, en busca de rehabilitar la polarización ideológica que había funcionado en la campaña electoral, aunque el panorama político era muy diferente y era ostensible para la opinión pública su aislamiento en el campo político. 
En medio de este proceso político se desarrollaron las elecciones de medio término, en las que se renovó la mitad de la Legislatura Local y de la representación porteña en la Cámara de Diputados de la Nación. En este proceso, la polémica y debilitada figura de Ibarra desapareció de la escena, lo que, además de profundizar su tendencia descendente, acentuó la volatilidad del campo político porteño. El proceso electoral de 2005 (para el que se presentaron 29 listas a diputados nacionales y 42 listas de candidatos a legisladores) estuvo protagonizado por tres nuevos alineamientos políticos, fruto de la fragmentación y reagrupamiento de los actores de 2003.

En primer lugar, Mauricio Macri volvió a presentarse como candidato ahora al frente de un nuevo sello partidario, Propuesta Republicana (PRO), una alianza entre CPC (coalición que agrupaba partidos conservadores con sectores peronistas y diversos cuadros políticos en disponibilidad) y Recrear (la fuerza liderada por el ex ministro Ricardo López Murphy, que en 2003 apoyó a Patricia Bullrich). En segundo lugar, el ARI se instaló en el rol de doble oposición, tanto al gobierno local como al gobierno nacional. Finalmente, el kirchnerismo presentó una lista de candidatos propios utilizando su sello electoral, el Frente para la Victoria (FPV, una alianza de cuatro partidos, que incluía al PJ).

La campaña electoral se desplazó hacia la discusión de la política nacional, donde los dos candidatos opositores intentaron instalarse como la principal oposición al kirchnerismo. El FPV, por su parte, debió enfrentarse a una campaña electoral difícil en un distrito complejo, asumiendo dos costos. En primer lugar, no podía respaldarse en una coalición progresista amplia ni en una figura cercana pero autónoma como la de Ibarra, que podía asegurarle apoyos en un distrito poco proclive a votar por candidatos peronistas. En segundo lugar, haber asumido la defensa del Jefe de Gobierno implicaba costos electorales que el kirchnerismo no estaba en condiciones de asumir en la coyuntura de 2005, cuando se desarrollaba una batalla electoral fundamental en la Provincia de Buenos Aires, donde se enfrentaba a la estructura del PJ. En este sentido, Aníbal Ibarra no sólo no cubrió ninguna de las expectativas que el kirchnerismo había depositado en la llamada transversalidad, sino que incluso consumía capital político en una coyuntura crítica.

El proceso electoral culminó con un contundente triunfo del macrismo, que se fortaleció en la Legislatura local y como opción verosímil en la política nacional. El ARI también se benefició del proceso, acrecentando sus bancas en la Legislatura porteña, mientras que el FPV quedó relegado, aunque sin mayores costos para el kirchnerismo, que se había impuesto en el resto de los distritos subnacionales. 
Una vez pasadas las elecciones, la debilidad de Ibarra ofreció la oportunidad para el avance del proceso político que terminó en la destitución. El proceso obligó a los fragmentados actores de la Legislatura a tomar postura, y casi la totalidad del arco político (desde izquierda hasta centroderecha) se aglutinó contra el mandatario local. Ibarra sólo fue sostenido por un puñado de legisladores propios o aliados y por el indisciplinado bloque kirchnerista, que no votó de manera uniforme en ninguna de las instancias que definieron la destitución. El comportamiento ambivalente del kirchnerismo obedeció a las contradicciones internas sobre cómo construir una coalición en el distrito, dadas las intenciones del vicejefe de gobierno, el peronista Jorge Telerman, de desplazar a Ibarra y convertirse en el nuevo vértice de una coalición progresista que incluyera al FPV.

\section{LAS ELECCIONES DE 2007 Y LA REORGANIZACIÓN DE LAS COALICIONES ELECTORALES}

El gobierno provisional de Jorge Telerman encontraba numerosas similitudes con la situación que atravesó Aníbal Ibarra luego de la crisis de 2001. Igual que Ibarra, Telerman ocupaba el gobierno sin un partido político o una coalición que lo sostuviera, habida cuenta de que tanto la alianza de gobierno como la coalición legislativa se habían disuelto con la crisis política e incluso antes. Y, del mismo modo que su antecesor, la necesidad de construir un actor partidario desde el vértice del poder estatal no sólo obedecía al requerimiento de la gobernabilidad, sino también a la ambición de construir un armado político competitivo para revalidarse electoralmente en el cargo

La incorporación de Telerman en la escena política porteña alteró el campo constituido en las elecciones de 2005, disputada por coaliciones electorales hegemonizadas por el PRO, el ARI y el FPV. Su visibilidad pública lo había instalado tempranamente como figura excluyente del proceso electoral, elemento que complementó con el uso estratégico de los recursos del ejecutivo porteño (especialmente la nominación de funcionarios públicos y la definición del calendario electoral), para construir una coalición electoral competitiva. Del mismo modo que Ibarra en 2003, Telerman intentó condensar bajo su figura un "arco progresista" autónomo de cualquier fuerza nacional.

Para ello, interpeló a una multitud de organizaciones partidarias, entre el oficialismo nacional y en la oposición. La articulación de estos actores en una opción electoral 
se implementó, en primer lugar, en la conformación del gabinete porteño, donde se encontraron referentes que venían del ibarrismo con funcionarios del FPV, del PS y de la UCR, o vinculados personalmente al candidato presidencial Roberto Lavagna. En un contexto donde ninguna fuerza política podía presentar candidaturas competitivas en todas las arenas electorales, Telerman se ofreció a los tres principales candidatos presidenciales como figura independiente capaz de garantizar el triunfo electoral, central para posicionarse ante la competencia nacional. Esta necesidad era más pronunciada entre los partidos de oposición, cuyas principales figuras habían construido su protagonismo exclusivamente en la arena porteña pero no disponían de candidatos relevantes propios.

Siguiendo el patrón que organizó la escena electoral cuatro años antes, Telerman combinó la convocatoria a los candidatos presidenciales que buscaban hacer pie en la Ciudad con el uso estratégico del calendario electoral para limitar sus alternativas y forzarlos a apoyar su candidatura. Adelantando las elecciones locales, dificultó la instalación de aquellos candidatos con menor reconocimiento público, y acortó los tiempos para que los actores partidarios definieran sus estrategias, lo que determinó la escena electoral posterior.

Como en 2003, el uso estratégico del calendario electoral determinó la conformación de los principales actores, y los candidatos al ejecutivo local señalados por los sondeos de opinión fueron los polos de atracción de fragmentos partidarios y los vértices de una oferta electoral organizada piramidalmente, donde dos o más listas legislativas competían entre sí y aportaban votos a la misma fórmula para el Ejecutivo. En torno de las candidaturas de Mauricio Macri, Daniel Filmus y Jorge Telerman se organizaron coaliciones partidarias y se reorganizó la fragmentada Legislatura $\mathrm{local}^{8}$. Dado que, a diferencia de las elecciones ejecutivas precedentes, sólo se renovaba la mitad del cuerpo legislativo, el número de listas en competencia se redujo sensiblemente.

La oferta política constituida (con 20 binomios al ejecutivo y 23 listas para legisladores locales) ubicaba al tope de las preferencias a un grupo de coaliciones (en algunos casos, coaliciones entre coaliciones) entre precandidatos autoproclamados y fragmentos de estructuras organizativas.

\footnotetext{
8 Se formaron tres grandes interbloques: uno macrista (de 22 legisladores), otro alineado tras la candidatura de Daniel Filmus (con 16 legisladores) y un tercero con el Jefe de Gobierno (con 15 legisladores).
} 
El proceso de negociación de candidaturas y la organización de las alianzas electorales fue abiertamente conflictivo, en algunos casos por la novedad y premura en la conformación de nuevos actores (como la candidatura de Jorge Telerman), en otros por las profundas divisiones internas entre coaliciones de casi nula institucionalización (Macri y Filmus). En esta coyuntura, la comunicación política no sólo jugó un rol para la presentación de los perfiles de los candidatos, sino que también fue vital para la formación de las alianzas electorales, incrementando o estrechando el margen de negociación de los diferentes actores involucrados, y realineando las lealtades electorales antes y después del cierre de las listas.

La nueva candidatura de Macri al ejecutivo porteño fue el resultado de tensiones internas del PRO, surgidas de su heterogeneidad organizacional, el adelantamiento del calendario electoral y la falta de figuras competitivas para disputar las principales arenas electorales. Como las otras terceras fuerzas del sistema político argentino, Macri había construido CPC como una serie concéntrica de alianzas. En 2003, con centro en un acotado círculo de cuadros políticos, había convocado a un fragmento del PJ porteño, a un grupo de partidos menores (Partido Federal, UCEDÉ y Partido Demócrata) y a otros cuadros independientes. En 2005, la anexión de RECREAR agregó una nueva capa a este entramado, y obligó al líder de CPC a convivir con el liderazgo de Ricardo López Murphy. Sin solidaridades cristalizadas en mecanismos internos, cada facción buscó imponer sus estrategias coalicionales, e impulsar o erosionar candidaturas.

La candidatura presidencial de Macri era promocionada por el círculo más cercano de consejeros del empresario y los responsables del armado del PRO a nivel nacional. Sin embargo, los sectores vinculados al armado local defendían la candidatura del ingeniero en la Ciudad, argumentando que sólo Macri podía garantizarles el crecimiento como fuerza política en el distrito. La negativa de estos sectores a apoyar a un candidato menos competitivo y la amenaza de dispersión del capital político acumulado, obligaron al ingeniero a resignar sus aspiraciones presidenciales y mantenerse en la disputa local. Para las legislativas, el PRO resolvió sus internas y logró conformar una única lista de candidatos que incluía a los siete partidos de la coalición, aunque sobrerrepresentaba a las facciones más cercanas a Macri.

En el FPV, la candidatura de Daniel Filmus también se definió en un contexto de fuerte división interna. Con los sucesivos fracasos que significaron la derrota electoral de 2005 y la destitución de Aníbal Ibarra, la convocatoria de Telerman a integrar una coalición electoral resultaba atractiva para sectores del PJ porteño, pero era vetada por el Presidente, que finalmente apoyó la candidatura del Ministro de Edu- 
cación, Daniel Filmus (quien hasta 2003 había sido ministro del gobierno de Ibarra en la Ciudad). A pesar de ser el ministro con mayor imagen positiva en el electorado, Filmus no era una figura reconocida por el electorado, y el adelantamiento del calendario electoral reducía sus chances de instalarse como un candidato competitivo, lo que dificultaba el alineamiento de las múltiples organizaciones kirchneristas con base en el distrito.

Con el objetivo de potenciar su figura, Filmus celebró una alianza con su ex jefe político, Aníbal Ibarra, quien, luego de su destitución abandonó sus filiaciones partidarias y fundó un nuevo sello, Diálogo por Buenos Aires (DBA), una coalición con otros referentes de la política porteña interesados en sumar bancas en la Legislatura y en la Cámara de Diputados. Al filo del cierre para la presentación de candidaturas, el FPV (que agrupaba a ocho partidos) y DBA (una alianza de seis etiquetas) presentaron al binomio Filmus-Heller para el ejecutivo local, y dos listas (una por cada coalición) para la Legislatura local.

Telerman, del mismo modo que Ibarra, había logrado alinear detrás de su candidatura al PS, a sectores del kirchnerismo e incluso a la UCR y otros partidos menores (como la Democracia Cristiana y el Partido Verde). Este colectivo había sido organizado en función de dos listas legislativas (una denominada Más Buenos Aires y otra Buenos Aires por Más), ante la imposibilidad de dirimir candidaturas entre partidos políticos, organizaciones sociales y cuadros políticos en disponibilidad. Con el adelantamiento del calendario electoral, Telerman logró, al filo del cierre de presentación de candidaturas, el alineamiento del ARI, el partido de Elisa Carrió. La nueva coalición (que agrupaba a once etiquetas) presentó dos listas de candidatos a la legislatura, una que reagrupaba a todo el conglomerado telermista y otra que representaba al armado de Carrió.

La alianza se produjo en un contexto de profunda división interna del ARI, dado que Carrió buscaba reorientar su partido como una alternativa liberal al kirchnerismo (rebautizada como Coalición Cívica), diluyendo el componente de centroizquierda que lo había caracterizado. Del otro lado, la alianza generó una crisis en la coalición telermista, que derivó en la progresiva salida de los sectores kirchneristas (que se pasaron a las filas de Filmus) y disolvió el perfil que Telerman intentaba instituir, factores que lo debilitaron profundamente en la arena electoral.

Esta lógica coalicional entre espacios políticos difícilmente identificables como partidos en el sentido tradicional del término (se trataba, en todo caso, de etiquetas que alineaban fragmentos organizacionales con liderazgos de popularidad), se reflejó en 
la campaña electoral. Los principales actores intentaron activar una identificación con el electorado a partir de la "sumatoria" de perfiles diversos, y al mismo tiempo diferenciarse de sus adversarios a partir de las deficiencias de sus socios o de la heterogeneidad de sus armados.

En esta lógica, tanto Filmus como Telerman intentaron ubicarse como herederos de la tradición progresista que había encarnado Ibarra en 2003. La disputa por la herencia progresista consumió sus esfuerzos de campaña, en la convicción de que la figura de Mauricio Macri resultaba expulsiva para la mayoría de los porteños y perdería en cualquier escenario de ballotage. Estas convicciones se revelaron injustificadas luego del holgado triunfo de Macri en la primera vuelta. Aunque debió transitar el ballotage (algo que no hubiera sucedido en otro contexto institucional), ello redundó en una victoria aún mayor: obtuvo, por primera vez en elecciones a Jefe de Gobierno, más de un millón de votos (60,94\%), frente al 39.06\% de Filmus-Heller.

La hegemonía macrista se amplificó en la distribución de bancas en la Legislatura local, debido a que los votos del PRO se concentraron en una única lista, mientras que los de Filmus y Telerman se dividieron entre las distintas listas que presentaron. De este modo, el macrismo accedió al gobierno porteño casi con mayoría absoluta, mientras que sus adversarios quedaron divididos y debilitados ante la opinión pública. Durante el período siguiente, el control del ejecutivo local fue la clave para sostener a la figura de Macri (y al PRO como alternativa política) a lo largo de las heterogéneas coyunturas que se presentaron. Durante los cuatro años siguientes se mantendrían las condiciones de baja institucionalización del sistema partidario, con la emergencia de nuevos liderazgos sociales y políticos, con la constitución y disolución de nuevas coaliciones, y con la restructuración del campo político a través de sucesivas coyunturas electorales y políticas. Sólo el PRO, con el liderazgo personalista de Macri desde el ejecutivo local, y el FPV, con el liderazgo personalista de Cristina Kirchner desde el ejecutivo nacional (y el sostenido apoyo a Daniel Filmus como figura representativa en la Ciudad), se mantuvieron en pie a través de un contexto político en permanente cambio.

\section{CONCLUSIONES}

En el presente artículo nos hemos preguntado por el fenómeno del crecimiento de las coaliciones partidarias en un contexto de colapso del sistema partidario y fragmentación de los partidos. Nuestra hipótesis apuntó a identificar un nuevo patrón 
de construcción partidaria, según el cual se articulan de manera circunstancial liderazgos populares en la opinión pública con fragmentos de estructuras territoriales y otros cuadros políticos disponibles.

A partir de estos argumentos, hemos descrito el proceso de implosión del sistema partidario porteño y su precaria reorganización en función de coaliciones políticas circunstanciales. Hemos repasado de qué modo se disolvió la relación entre tradiciones políticas y organizaciones partidarias, describiendo la emergencia y evolución de nuevas etiquetas partidarias, más eficaces en atraer la atención de la ciudadanía a partir de cortar lazos con las organizaciones partidarias tradicionales.

Estos nuevos armados políticos han recurrido a la formación de coaliciones selectivas en distintas arenas (electoral, legislativa, de gobierno) para poder competir en elecciones y formular políticas públicas. Estas coaliciones se caracterizan por su baja institucionalización y su morfología heterogénea, donde conviven organizaciones sociales sin peso electoral con líderes personalistas y etiquetas partidarias poco representativas. Dichas alianzas parecen conformarse y restructurarse en función de dos tipos de coyunturas.

En primer lugar, los procesos electorales generan contextos de profunda reorganización de las solidaridades partidarias, en tanto la emergencia de candidatos competitivos funciona como polos de atracción para una pluralidad de actores. En este contexto, la normativa electoral aparece como una clave para generar una escena electoral específica, y los mandatarios locales utilizan sus prerrogativas para estructurar el campo político. Del mismo modo, el campo de la comunicación política no sólo contribuye a la definición de los perfiles políticos durante la campaña electoral, sino que incide en la conformación misma de las coaliciones que compiten en elecciones.

En segundo lugar, coyunturas políticas que alcanzan una intensidad crítica (a partir de la movilización social o de la división política que generan ciertos fenómenos) también tienen efectos en la reconfiguración del campo político. Hemos analizado el caso del juicio político al jefe de gobierno, donde la movilización social derivó en la reorganización de la coalición parlamentaria y de gobierno de Aníbal Ibarra; modificando por completo la escena política de las elecciones de 2005 y 2007. En estas coyunturas, el campo de la comunicación política también resulta vital, posicionando discursos y favoreciendo o dificultando la conformación de actores sociales y partidarios. 
Finalmente, hemos señalado cómo el uso de los recursos del gobierno local facilita el posicionamiento y la continuidad de las coaliciones políticas, en un contexto de baja institucionalidad de todos los actores y de alta volatilidad del electorado. Los casos de la destitución de Ibarra y de la derrota de Telerman en las elecciones de 2007, sin embargo, demuestran los límites del recurso a los resortes del ejecutivo. En este sentido, la construcción de una identidad que cohesione coaliciones partidarias heterogéneas y garanticen el vínculo entre las élites políticas y la ciudadanía es una dimensión ineludible en un contexto de desagregación partidaria y fluctuación electoral. Es sólo a través de los procesos de diferenciación política que los acontecimientos públicos adquieren significación política, y que los individuos pueden reconocerse como partícipes de un proceso democrático, en una dimensión que va más allá del puro pragmatismo con el que se caracteriza a las redes políticas.

\section{REFERENCIAS BIBLIOGRÁFICAS}

Bril Mascarenhas, Tomás (2007). "El colapso del sistema partidario de la ciudad de Buenos Aires. Una herencia de la crisis argentina de 2001-2002”. Desarrollo Económico, Vol. 47, No 187 , pp. 367-400.

Calvo, Ernesto y Marcelo Escolar (2005). La nueva política de partidos en la Argentina. Buenos Aires: Prometeo.

Cheresky, Isidoro (comp.) (2006). La política después de los partidos. Buenos Aires: Prometeo.

Gibson, Edward y Julieta Suárez-Cao (2010). "Federalized Party Systems and Subnational Party Competition: Theory and an Empirical Application to Argentina" Comparative Politics.Vol. 43. ํㅜ 1.

Leiras, Marcelo (2007). Todos los caballos del rey. Buenos Aires: Prometeo.

Mair, Peter (1997). Party system change: Approaches and interpretations. Oxford: Clarendon Press.

Mair, Peter (2005). Democracy beyond parties. Irvine: Center for the Study of Democracy.

Malamud,Andrés (2004). "El bipartidismo argentino: evidencias y razones de una persistencia (1983-2003)”. Revista Colección No15, pp. 13-43.

Manin, Bernard (1998). Los Principios del Gobierno Representativo. Madrid: Alianza.

Mauro, Sebastián (2007). "Mutación, crisis, recomposición y otra vez crisis de la representación política en la ciudad de Buenos Aires. Los avatares del signo progresista". Argumentos, Revista de crítica social $\mathrm{N}^{\circ} 8$. 
Mauro, Sebastián (2011). Representación e identificaciones políticas en tiempos de solidaridades inestables (Ciudad de Buenos Aires, 2001-2007), tesis doctoral defendida en la Facultad de Ciencias Sociales, Universidad de Buenos Aires.

Scherlis, Gerardo (2009). “El partido estatal estratárquico de redes. Apuntes sobre organización política en la era de los partidos no representativos”, en Cheresky, Isidoro (comp.). Las urnas y la desconfianza ciudadana en la democracia argentina. Rosario: Homo Sapiens.

Torre, Juan Carlos (2003). "Los huérfanos de la política de partidos”. Desarrollo Económico,Vol. $42, \mathrm{~N}^{\circ} 168$, pp. 647-665.

Vommaro, Gabriel (2007). Lo que quiere la gente. Sondeos de opinión y espacio de la comunicación politica en Argentina. Buenos Aires: Prometeo.

Recibido: 29 enero 2012 / Aprobado: 26 abril 2012 